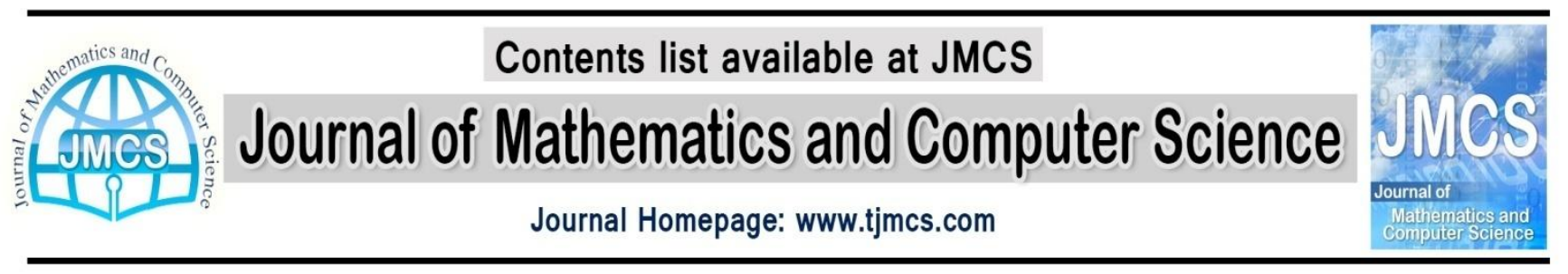

\title{
The analytical solution of singularly perturbed boundary value problems
}

\author{
S. Gh. Hosseini ${ }^{a}$, S. M. Hosseini ${ }^{b}$, M. Heydari ${ }^{c}$, M. Amini $^{d}$ \\ ${ }^{a}$ Department of Mathematics, Ashkezar Branch, Islamic Azad University, Ashkezar, Iran \\ ${ }^{b}$ Department of Mathematics, College of Science, Shahrekord Branch, Islamic Azad University, \\ Shahrekord, Iran \\ ${ }^{c}$ Department of Mathematics, Yazd Branch, Islamic Azad University, Yazd, Iran \\ ${ }^{d}$ Department of Computer Engineereing, Khatam Center, Islamic Azad University, Yazd, Iran
}

Article history:

ghasem602@yahoo.com (Corresponding Author: S. Gh. Hosseini)

Received January 2014

Accepted March 2014

Available online March 2014

\begin{abstract}
In this paper, we present an algorithm for approximating numerical solution of singularly perturbed boundary value problems by means of homotopy analysis and tau Bernestein polynomial method. The method is tested for several problems and the results demonstrate reliability and efficiency of the method.

Keywords: Singularly perturbed problems; Boundary value problems; Homotopy analysis method; Galerkin's method; Bernstein polynomials.
\end{abstract}

\section{Introduction}

We consider a class of singularly perturbed two-point singular boundary value problem of the form

$$
\varepsilon y^{\prime \prime}(x)+f\left(x, y, y^{\prime}\right)=0, \quad x \in[0,1],
$$

subject to the boundary conditions

$$
y(0)=\alpha, \quad y(1)=\beta, \quad \alpha, \beta \in \Re
$$

where $\varepsilon$ is a small positive parameter. 
In general, as $\varepsilon$ tends to zero, the solution $y(x)$ may exhibit exponential boundary layers at left-end of the interval $[0,1]$.

These problems arise frequently in many areas of science and engineering such as heat transfer problem with large Peclet numbers, Navier-Stokes flows with large Reynolds numbers, chemical reactor theory, aerodynamics, reaction-diffusion process, quantum mechanics, optimal control etc [6]. Due to the variation in the width of the layer with respect to the small perturbation parameter $\varepsilon$. Several difficulties are experienced in solving the singular perturbation problems using standard numerical methods.

Several numerical methods have been developed for the numerical solution of singularly perturbed boundary value problems, in particular to the problems having the boundary layers at one or both ends of the interval. Boglaev [4], Schatz and Wahlbin [24] used the finite element technique to solve such types of problems. Miller [14] gave sufficient conditions for the first-order uniform convergence of three-point difference scheme. Cen et al. [5] presented hybrid finite difference scheme with Shishkin mesh for solving a system of singularly perturbed initial value problems. While Stojanovic [25] gave an optimal difference scheme by considering the quadratic interpolating splines instead of piecewise constants on each subinterval $\left[x_{i-1}, x_{i}\right]$ as an approximation for the coefficient $f(x)$. Surla and Jerkovic [26] considered the spline collocation method for the solution of singularly perturbed boundary value problems. Rao and Kumar [17] gave an optimal B-spline collocation method for solving singularly perturbed boundary value problems. Loghmani and Ahmadinia [16] develop a numerical technique for singularly perturbed boundary value problems using B-spline functions and least square method. Dua and Kong [6] used the new Liouville-Green transform to solve a singularly perturbed second-order ordinary differential equation. Attili [2] used Pade approximation to obtain the solution of singularly perturbed two point boundary value problems.

The concept of replacing singularly perturbed two-point boundary value problem by an initial value problem is presented by Reddy et al. [12], [21], [22]. Reddy and Chakravarthy [20] have extended boundary value technique to solve general singularly perturbed two-point boundary value problems using trapezoidal formula integration in the forward direction with left-layer boundary problems and in backward direction with right-layer boundary problems, and both formulas for interior or two boundary layers, where, their method is iterative on the deviating argument.

\section{Bernestein homotopy method}

\subsection{Bernstein polynomials}

The Bernstein polynomials of degree $K$ are defined on the interval $[0,1]$ as [29]

$$
B_{i, K}(x)=\left(\begin{array}{c}
K \\
i
\end{array}\right) x^{i}(1-x)^{K-i}, 0 \leq i \leq n,
$$

where the binomial coefficients are given by

$$
\left(\begin{array}{l}
K \\
i
\end{array}\right)=\frac{K !}{i !(K-i) !}
$$


These Bernstein polynomials form a basis on [0,1]. There are $K+1, K$ th-degree polynomials. For convenience, we set $B_{i, K}(x)=0$ if $i<0$ or $i>K$. Moreover, the recursive definition for the Bernstein polynomials over the interval $[0,1]$ is as follows:

$$
B_{i, K}(x)=(x-1) B_{i, K-1}(x)+x B_{i-1, K-1}(x) .
$$

It can be readily shown that the sum of all Bernstein polynomials of degree $K$ is the constant 1 , that is, $\sum_{i=0}^{K} B_{i, K}(x)=1$, and for all $i=0,1, \cdots, K$ and all $x$ in $[0,1]$, we have $B_{i, K}(x) \geq 0$. Also, we have

$$
B_{i, K}(0)= \begin{cases}1 & i=0 \\ 0 & i \neq 0\end{cases}
$$

and

$$
B_{i, K}(1)= \begin{cases}1 & i=K \\ 0 & i \neq K\end{cases}
$$

The Bernstein polynomials are widely used for numerical solutions of differential, integral, and integrodifferential equations [27]-[39].

\subsection{Homotopy analysis method}

In 1992 Liao [40], used the basic idea of homotopy in topology to proposed an analytical technique for solving non-linear problems. This technique, namely homotopy analysis method [41]. The homotopy analysis method and its modifications have been efficiently employed to solve a wide range of nonlinear problems in applied sciences [41]-[62].

In this section, the homotopy analysis method is used to give series solution of the (1) with boundary conditions (2). We define an auxiliary linear operator $L$ by

$$
L[\phi(x, \lambda ; q)]=\varepsilon \phi^{\prime \prime}(x ; q)+\phi^{\prime}(x ; q)
$$

with the property

$$
L\left[C_{1}+C_{2} e^{-\frac{x}{\varepsilon}}\right]=0
$$

where $C_{1}$ and $C_{2}$ are constants.

We define a nonlinear operator in the form:

$$
N[\phi(x ; q)]=\varepsilon \phi^{\prime \prime}(x ; q)+f\left(x, \phi(x ; q), \phi^{\prime}(x ; q)\right) .
$$

Using this operator, we can construct the zeroth-order deformation equation as

$$
(1-q) L\left[\phi(x ; q)-y_{0}(x)\right]=q \hbar N[\phi(x ; q)]
$$

where $\hbar \neq 0$ is an auxiliary parameter and $q \in[0,1]$ is an embedding parameter. The boundary conditions for Eq. (11) are 


$$
\phi(0 ; q)=\alpha, \quad \phi(1 ; q)=\beta
$$

When the parameter $q$ increases from 0 to 1 , the solution $\phi(x ; q)$ varies from $y_{0}(x)$ to $y(x)$. If this continuous variation is smooth enough, the Maclaurin series with respect to $q$ can be constructed for $\phi(x ; q)$, and further, if this series is convergent at $q=1$, we have

$$
y(x)=y_{0}(x)+\sum_{i=1}^{\infty} y_{i}(x)=\sum_{i=0}^{\infty} \varphi_{i}(x, \hbar)
$$

where

$$
y_{i}(x)=\left.\frac{1}{i !} \frac{\partial^{i} \phi(x ; q)}{\partial q^{i}}\right|_{q=0}
$$

For the $m$ th-order deformation equation, we differentiate Eqs. (11)-(12) $m$ times with respect to $q$, divide by $m$ ! and then set $q=0$. The resulting $m$ th-order deformation equation is

$$
L\left[y_{m}(x)-\chi_{m} y_{m-1}(x)\right]=\hbar R_{m}(x)
$$

where

$$
\chi_{m}= \begin{cases}0, & m \leq 1 \\ 1, & m>1\end{cases}
$$

and

$$
R_{m}=\left.\frac{1}{m !} \frac{\partial^{m} N[\phi(x ; q)]}{\partial q^{m}}\right|_{q=0}
$$

with the following boundary conditions

$$
y_{m}(0)=y_{m}(1)=0
$$

\subsection{Tau Bernestein homotopy method}

In this subsection, we use the tau Bernestein method to obtain the solution of the first several Eq. (15) with boundary condition (18) and find that the $M$ th-order approximation of the numerical solution (1). The tau approach is a modification of the Galerkin method that is applicable to problems with non periodic boundary conditions $[63,64]$. For each $m=1,2, \cdots, M$ and an arbitrary natural number $\mathrm{K}$, we suppose that the approximate solution $y_{m}(x)$ is as follows:

$$
y_{m}(x)=\sum_{i=0}^{K} y_{m, i} B_{i, K}(x),
$$

and the residual function associated to the $m$ th-order deformation equation (15) is

$$
R E S y_{m}(x)=L\left[y_{m}(x)\right]-\chi_{m} L\left[y_{m-1}(x)\right]-\hbar R_{m}(x)
$$

By imposing the boundary conditions (18), we have

$$
\sum_{i=0}^{K} y_{m, i} B_{i, K}(0)=0, \sum_{i=0}^{K} y_{m, i} B_{i, K}(1)=0 .
$$

By using (6) and (7), we obtain 


$$
y_{m, 0}=0, y_{m, K}=0 .
$$

In tau method we get the inner product of Eq. (20) with $B_{S, K}(x)$ :

$$
\left\langle R E S y_{m}(x), B_{s, K}(x)\right\rangle=0, s=0,1, \cdots, K-2,
$$

where $\langle f, g\rangle=\int_{0}^{1} f(x) g(x) d x$. From the above equations, a linear system $A Y_{m}=\chi_{m} A Y_{m-1}+b_{m}$ is resulted, where

$$
\begin{aligned}
& a_{i, j}=\left\langle L\left(B_{j, k}(x)\right), B_{i-1, K}(x)\right\rangle=\varepsilon \int_{0}^{1} B_{j, k}^{\prime \prime}(x) B_{i-1, K}(x) d x \\
& +\int_{0}^{1} B_{j, k}^{\prime}(x) B_{i-1, K}(x) d x, \quad i, j=1,2, \cdots, K-1, \\
& \left(b_{m}\right)_{i}=\hbar\left\langle R_{m}(x), B_{i-1, K}(x)\right\rangle=\int_{0}^{1} R_{m}(x) B_{i-1, K}(x) d x, \quad i=1, \cdots, K-1, \\
& Y_{m}=\left(y_{m, 1}, y_{m, 2}, \cdots, y_{m, K-1}\right)^{T} .
\end{aligned}
$$

\subsection{Algorithm of presented method}

The presented method in subsection (2.3) can be done by using the following algorithm:

\section{Algorithm 1:}

Step 1. Set $y_{0}(x)=(\beta-\alpha) x+\alpha$.

Step 2. Calculate the matrix $A$, by applying the (24) and then compute $A^{-1}$.

Step 3. Compute the vector $b_{1}$ by applying the (25) and set $Y_{1}=A^{-1} b_{1}$ and $y_{1}(x)=$ $Y_{1}^{T} \phi(x)$, where

$$
\phi(x)=\left(B_{1, K}(x), B_{2, K}(x), \cdots, B_{K-1, K}(x)\right)^{T} .
$$

Step 4. For $m$ from 2 to $M$ do

Compute the vector $b_{m}$ by applying the (25) and set $Y_{m}=Y_{m-1}+A^{-1} b_{m}$ and $y_{m}(x)=$ $Y_{m}^{T} \phi(x)$,

End do.

Step 5. Set

$$
\Phi_{M, K}(x)=\sum_{i=0}^{M} y_{i}(x)
$$

as the approximate of the exact solution Eq. (1).

\subsection{Convergence of the solution}

From the section 3.3 in [41], as long as the series (27) is convergent, it should converge to a solution of Eq. (1). As pointed out by Liao [41], the convergence of this series and the rate of it depend upon the value of the auxiliary parameter $\hbar$. In general, the range of proper values of auxiliary parameters $\hbar$ is 
obtain by plotting the so called $\hbar$-curve. As pointed out by Liao [41], the valid region of $\hbar$ correspond to the line segments nearly parallel to the horizontal axis in $\hbar$-curve.

\section{Test problem}

In this section, we demonstrate the effectiveness of the presented algorithm by applied it to two nonlinear singular perturbed boundary value problems. The algorithm is performed by Maple 15 with 128 digits precision.

Example 3.1 Consider the following nonlinear singular perturbed boundary value problem:

$$
\varepsilon y^{\prime \prime}(x)+y^{\prime}(x)-y^{2}(x)=0
$$

with boundary conditions

$$
y(0)=1, \quad y(1)=1
$$

Solution: The problem (3.1) solved by using algorithm 1 for $K=50$ and $M=15$. In order to find the range of admissible values of $\hbar, \hbar$-curve of $y^{\prime}(0)$ obtained by the Alg. 1 is plotted in Fig. (1) for $\varepsilon=1,2^{-3}, 2^{-5}$. The residual error of Eq. (3.1) is plotted in Fig. (2) for different values of $\varepsilon$. Fig. (3) gives a comparison between the present method results and the numerical method. We can clearly observe from Fig. (3) that the solutions obtained by the proposed method are in good agreement with the numerical solutions.

Example 3.2 Consider the following nonlinear singular perturbed boundary value problem:

$$
\varepsilon y^{\prime \prime}(x)+\left(1-\frac{x}{2}\right) y^{\prime}(x)-\frac{1}{2} y^{2}(x)=0
$$

with boundary conditions

$$
y(0)=0, \quad y(1)=1
$$

Solution: The problem (3.2) solved by using algorithm 1 for $K=50$ and $M=10$. In order to find the range of admissible values of $\hbar, \hbar$ - curve of $y^{\prime}(0)$ obtained by the Alg. 1 is plotted in Fig. (4) for $\varepsilon=1,2^{-3}, 2^{-5}$. The residual error of Eq. (3.2) is plotted in Fig. (5) for different values of $\varepsilon$. Fig. (6) gives a comparison between the present method results and the numerical method. We can clearly observe from Fig. (6) that the solutions obtained by the proposed method are in good agreement with the numerical solutions. 

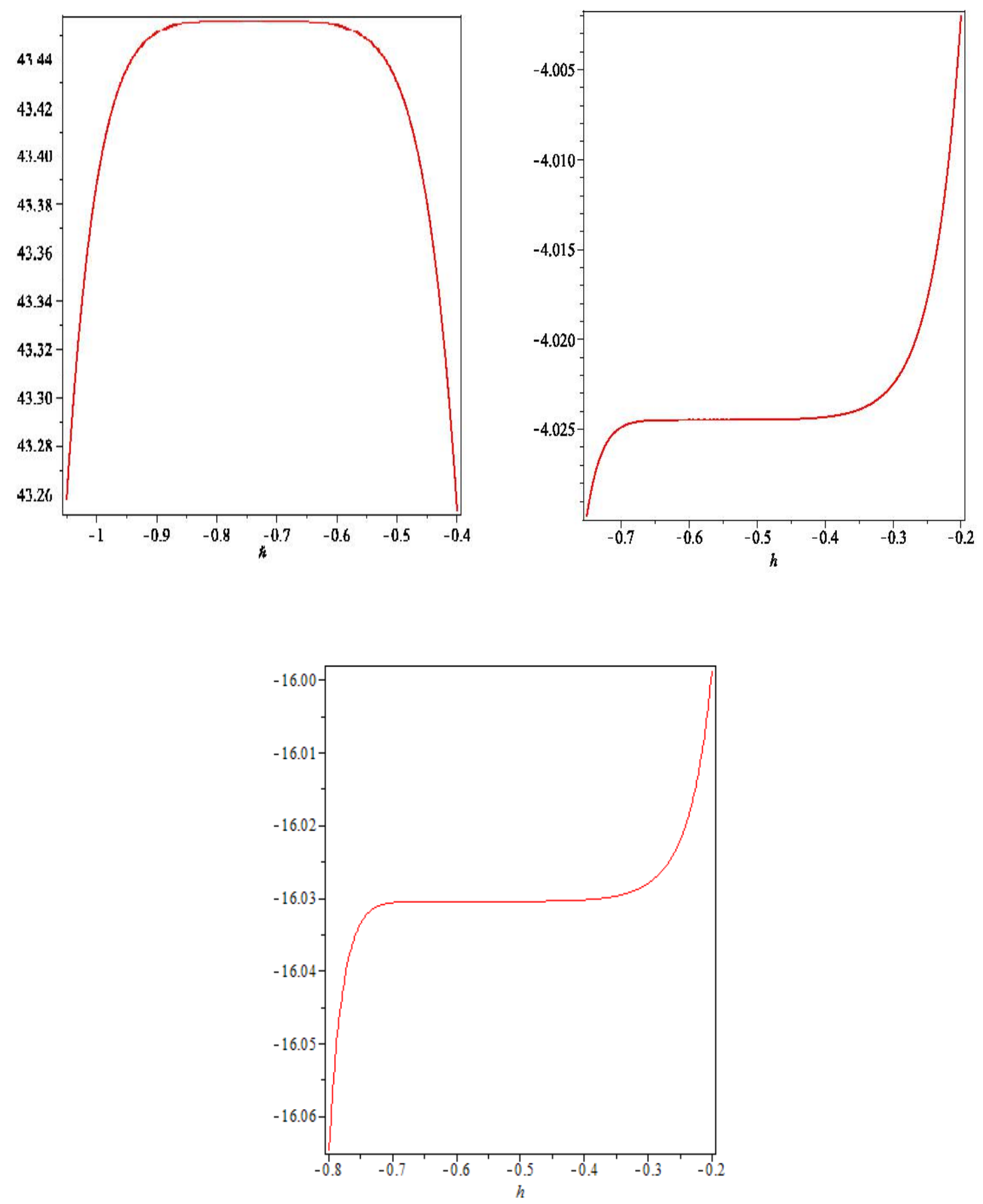

Figure 1: The $\hbar$-curves of $y^{\prime}(0)$ obtained by Alg. 1, when $K=50$ and $M=15$ for Ex. (3.1): left $(\varepsilon=1)$, right $\left(\varepsilon=2^{-3}\right)$ and bottom $\left(\varepsilon=2^{-5}\right)$ 

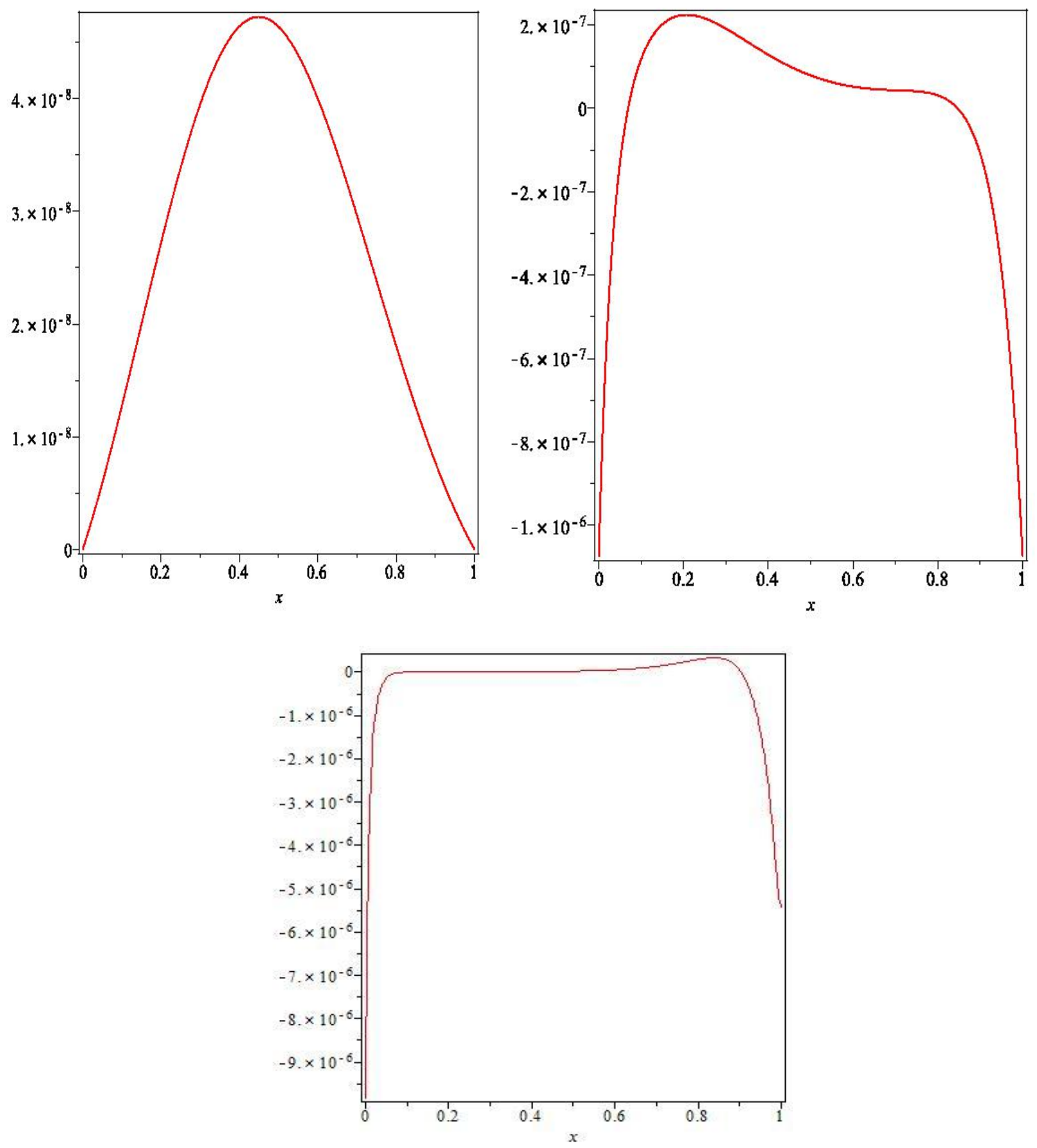

Figure 2: The residual error of Eq. (3.1) obtained by Alg. 1, when $K=50$ and $M=15$ for Ex. (3.1): left $(\varepsilon=1)$, right $\left(\varepsilon=2^{-3}\right)$ and bottom $\left(\varepsilon=2^{-5}\right)$. 


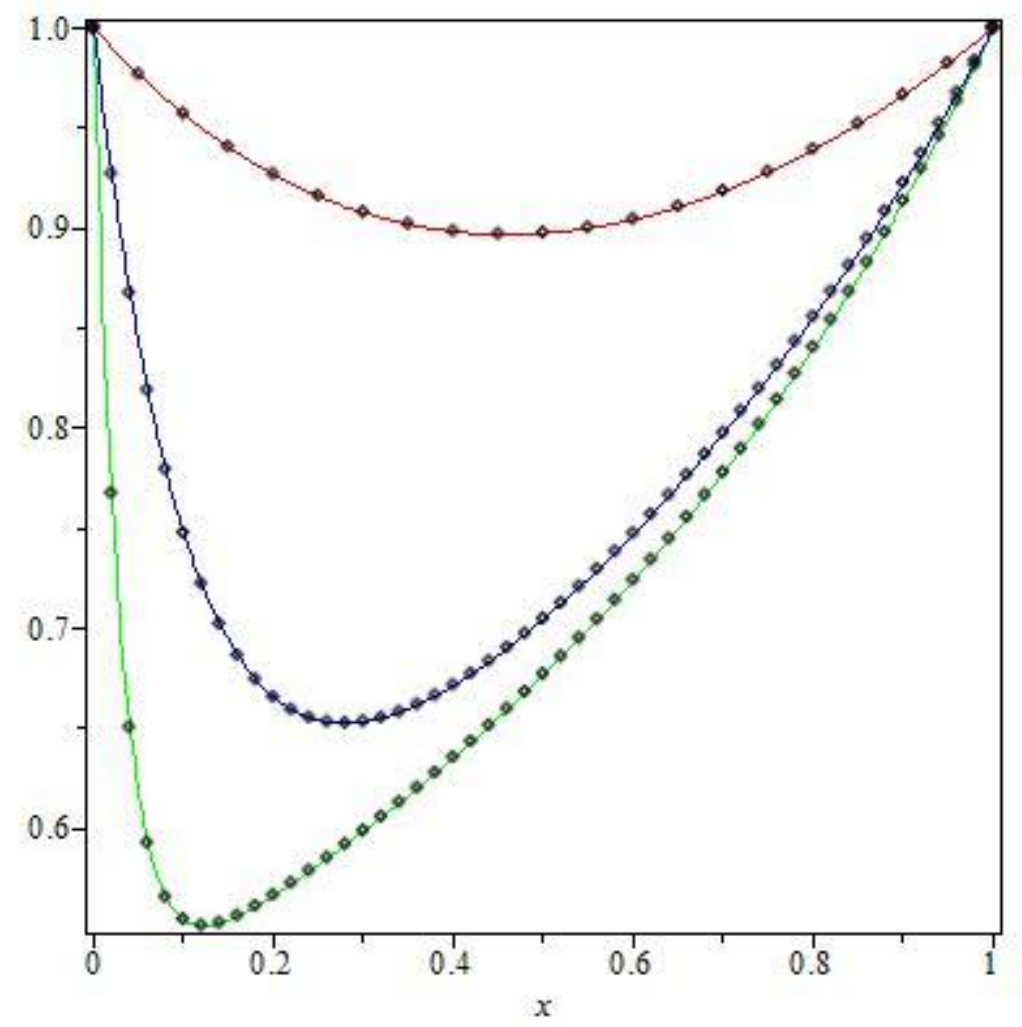

Figure 3: Plots of solution Eq. (3.1) obtained by Alg. 1, when $K=50$ and $M=15$ for Ex. (3.1): red $(\varepsilon=1)$, blue $\left(\varepsilon=2^{-3}\right)$ and green $\left(\varepsilon=2^{-5}\right)$; Circle: numerical solutions.

\section{Conclusions}

In this paper, the singularly perturbed two-point boundary layer problems have been considered by means of the homotopy analysis technique and tau Bernesetin method. The success of the method has later been tested by applying it to several singularly perturbed cases taken from the literature. The presented approach has clearly shown its advantage over the recently introduced conventional numerical methods for the singularly perturbed boundary value problems.

\section{References}

[1] A. Andargie, Y.N. Reddy, Fitted fourth-order tridiagonal finite difference method for singular perturbation problems, Appl. Math. Comp. 192 (2007) 90-100.

[2] B.S. Attili, Numerical treatment of singularly perturbed two point boundary value problems exhibiting boundary layers, Commun. Nonlinear Sci. Numer. Simulat. (In Press).

[3] C.M. Bender, S.A. Orszag, Advanced Mathematical Methods for Scientists and Engineers, McGrawHill, New York, 1978.

[4] I.P. Boglaev, A variational difference scheme for a boundary value problem with a small parameter multiplying the highest derivative, Zh. Vychisl. Mat. i Mat. Fiz. 21 (1981) 887-896. 

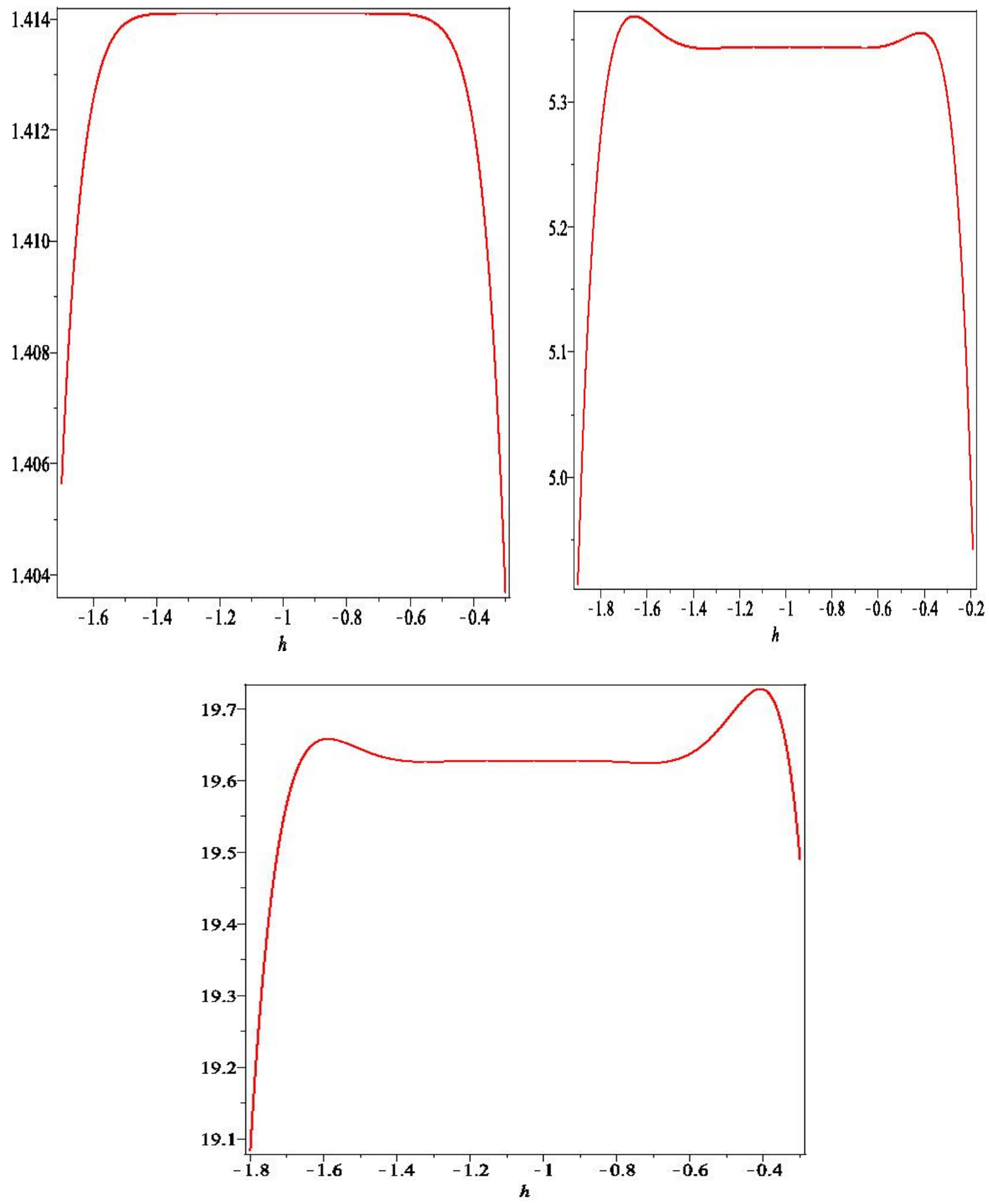

Figure 4: The $\hbar$-curves of $y^{\prime}(0)$ obtained by Alg. 1, when $K=50$ and $M=10$ for Ex. (3.1): left $(\varepsilon=1)$, right $\left(\varepsilon=2^{-3}\right)$ and bottom $\left(\varepsilon=2^{-5}\right)$. 

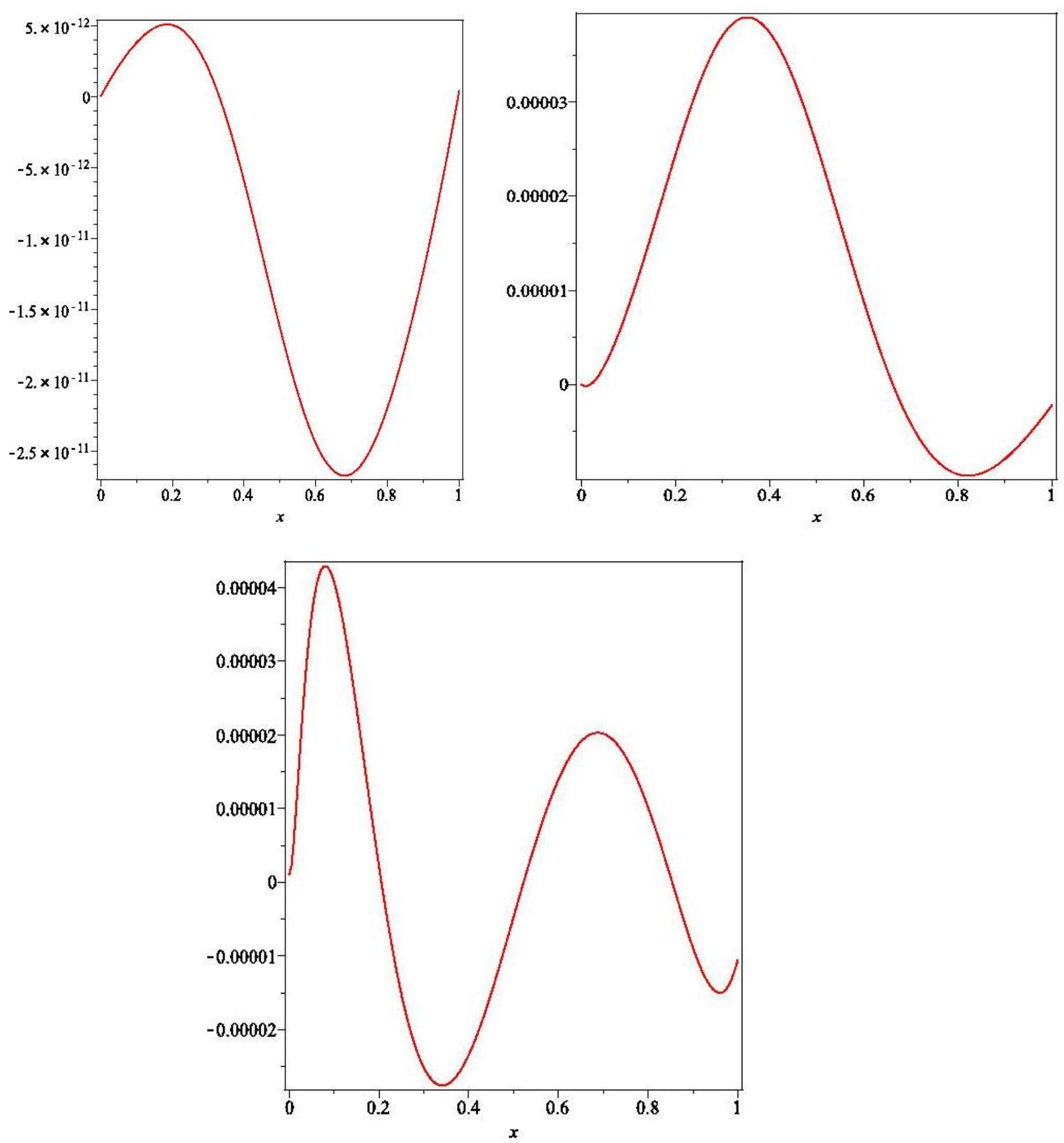

Figure 5: The residual error of Eq. (3.1) obtained by Alg. 1, when $K=50$ and $M=10$ for Ex. (3.2): left $(\varepsilon=1)$, right $\left(\varepsilon=2^{-3}\right)$ and bottom $\left(\varepsilon=2^{-5}\right)$. 


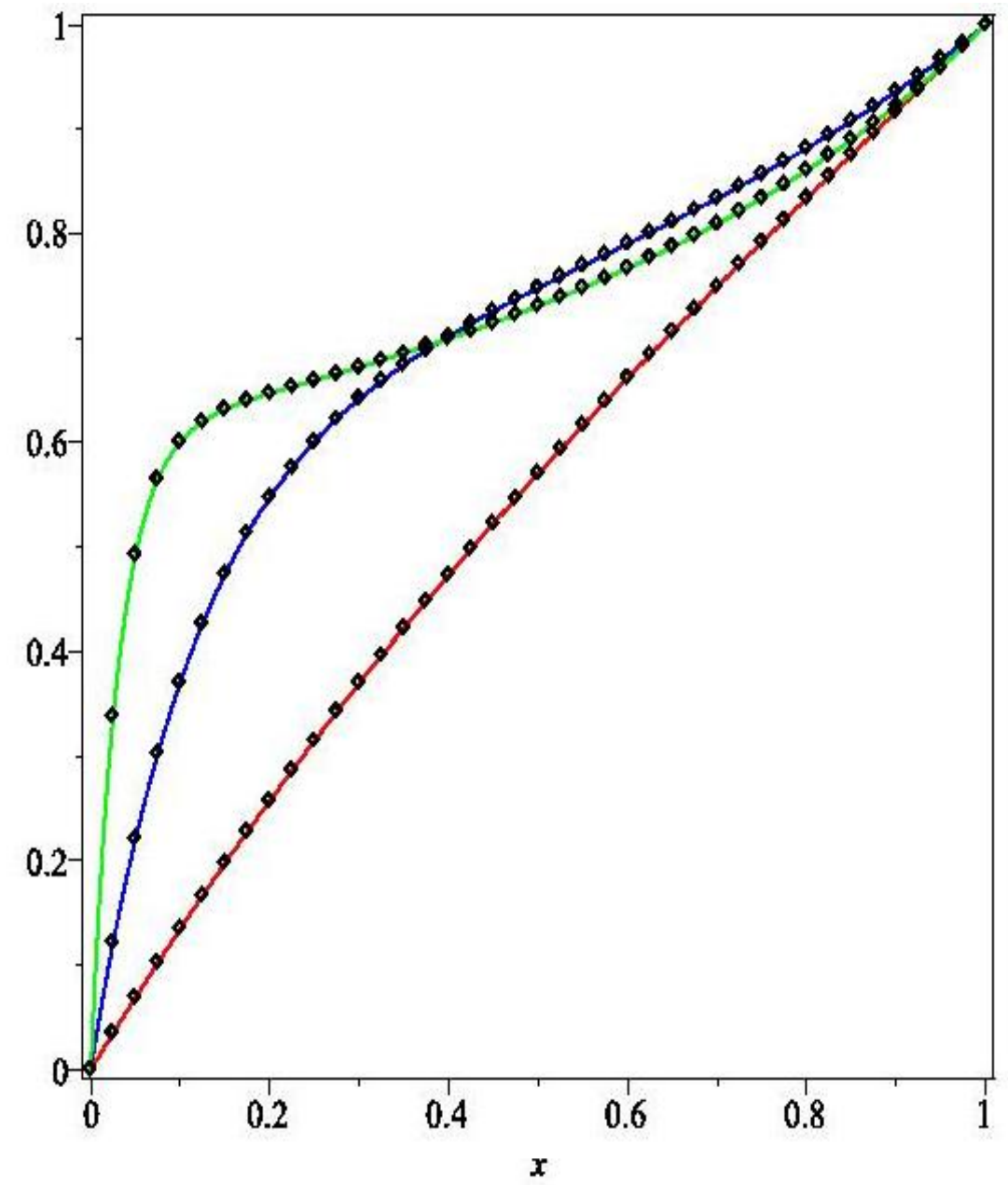

Figure 6: Plots of solution Eq. (3.1) obtained by Alg. 1, when $K=50$ and $M=10$ for Ex. (3.2): red $(\varepsilon=1)$, blue $\left(\varepsilon=2^{-3}\right)$ and green $\left(\varepsilon=2^{-5}\right)$; Circle: numerical solutions.

[5] Zh. Cen, A. Xu, A. Le, A second-order hybrid finite difference scheme for a system of singularly perturbed initial value problems, J. Comp. Appl. Math. 234 (2010) 3445-3457.

[6] Z. Dua, L. Kong, Asymptotic solutions of singularly perturbed second-order differential equations and application to multi-point boundary value problems, Appl. Math. Letters 23 (2010) 980-983.

[7] J.H. He, Homotopy perturbation technique, Comput. Methods Appl. Mech. Eng. 178 (1999) 257-262.

[8] J.H. He, Approximate analytical solution of Blasius equation, Commum. Nonlinear Sci. Numer. Simul. 3 (1998) 260-263.

[9] J.H. He, A coupling method of homotopy technique and perturbation technique for nonlinear problems, Internat. J. NonLinear Mech. 35 (1) (2000) 37-43. 
[10] J.H. He, A simple perturbation approach to Blasius equation, Appl. Math. Comput. 140 (2003) 217222.

[11] H.M. Habib, E.R. El-zahar, A new algorithm for general singularly perturbed two-point boundray value problems, Advances in Differential Equations and Control Processes, 1 (2008) 1-21.

[12] M.K. Kadalbajoo, Y.N. Reddy, Initial-value technique for a class of nonlinear singular perturbation problems, J. Optim. Theo. Appl. 53 (1987) 395-406.

[13] J. Kevorkian, J.D. Cole, Perturbation Methods in Applied Mathematics, Springer-Verlag, New York, 1981.

[14] J.J.H. Miller, On the convergence uniformly in e of difference schemes for a two point boundary singular perturbation problem, In Numerical analysis of singular perturbation problems (Proc. Conf. Math. Inst. Catholic Univ. Nijmegen 1978) Academic Press, London 1979 467-474.

[15] J. Mohapatraa, S. Natesan, Parameter-uniform numerical method for global solution and global normalized flux of singularly perturbed boundary value problems using grid equidistribution, Comput. Math. Appl. 60 (2010) 1924-1939.

[16] G.B. Loghmani, M. Ahmadinia, Numerical Solution of Singularly Perturbed Boundary Value Problems Based on Optimal Control Strategy, Acta Appl. Math. 112 (2010) 69-78.

[17] S.C.S. Rao, M. Kumar, Optimal B-spline collocation method for self-adjoint singularly perturbed boundary value problems, Appl. Math. Comput. 188 (2007) 749-761.

[18] A. Ramesh, N. Ramanujam, An asymptotic finite element method for singularly perturbed third and fourth order ordinary differential equations with discontinuous source term, Appl. Math. Comp. 191 (2007) 372-380.

[19] J. Rashidinia, R. Mohammadi, M. Ghasemi, Cubic spline solution of singularly perturbed boundary value problems with significant first derivatives, Appl. Math. Comp. 190 (2007) 1762-1766.

[20] Y.N. Reddy, P.P. Chakravarthy, Numerical integration method for general singularly perturbed two point boundary value problems, Appl. Math. Comput. 133 (2002) 351-373.

[21] Y.N. Reddy, P.P. Chakravarthy, An initial-value approach for solving singular perturbed two-point boundary value problems, Appl. Math. Comput. 155 (2004) 95-110.

[22] Y.N. Reddy, P.P. Chakravarthy, An exponentially fitted finite difference method for singular perturbation problems, Appl. Math. Comput. 154 (2004) 83-101.

[23] H.J. Reinhardt, Singular perturbations of difference methods for linear ordinary differential equations, Appl. Anal. 10 (1980), 53-70.

[24] A.H. Schatz, L. B.Wahlbin, On the finite element method for singularly perturbed reaction-diffusion problems in two and one dimensions, Math. Comput. 40 (1983) 47-89. 
[25] M. Stojanovic, Spline collocation method for singular perturbation problem, Glas. Mat. Ser. III 37 (2002) 393-403.

[26] K. Surla, V. Jerkovic, Some possibilities of applying spline collocations to singular perturbation problems, In Numerical methods and approximation theory, II Univ. Novi Sad 10 (1985) 19-25.

[27] D.D. Bhatta, M.I. Bhatti, Numerical solution of KdV equation using modified Bernstein polynomials, Appl. Math. Comput. 174 (2006) 1255-1268 .

[28] B.N. Mandal, S. Bhattacharya, Numerical solution of some classes of integral equations using Bernstein polynomials, Appl. Math. Comput. 190 (2007) 1707-1716.

[29] M.I. Bhatti, P. Bracken, Solutions of differential equations in a Bernstein polynomial basis, J. Comput. Appl. Math. 205 (2007) 272-280.

[30] S. Bhattacharya, B.N. Mandal, Use of Bernstein polynomials in numerical solutions of Volterra integral equations, Appl. Math. Sci. 2 (2008) 1773-1787.

[31] A. Chakrabarti, S.C. Martha, Approximate solutions of Fredholm integral equations of the second kind, Appl. Math. Comput. 211 (2009) 459-466.

[32] A.K. Singh, V.K. Singh, O.P. Singh, The Bernstein operational matrix of integration, Appl. Math. Sci. 3 (2009) 2427-2436.

[33] S.A. Yousefi, M. Behroozifar, Operational matrices of Bernstein polynomials and their applications, Int. J. Syst. Sci. 41 (2010) 709-716.

[34] S.A. Yousefi, M. Behroozifar, M. Dehghan, The operational matrices of Bernstein polynomials for solving the parabolic equation subject to specification of the mass, J. Comput. Appl. Math. 235 (2011) 5272-5283.

[35] E.H. Doha, A.H. Bhrawy, M.A. Saker, On the derivatives of Bernstein polynomials: An application for the solution of high even-order differential equations, Bound. Value. Probl. doi:10.1155/2011/829543.

[36] E.H. Doha, A.H. Bhrawy, M.A. Saker, Integrals of Bernstein polynomials: An application for the solution of high even-order differential equations, Appl. Math. Lett. 24 (2011) 559-565.

[37] S.A. Yousefi ,M. Behroozifar, M. Dehghan, Numerical solution of the nonlinear age-structured population models by using the operational matrices of Bernstein polynomials, Appl. Math. Model. 36 (2012) 945-963.

[38] K. Maleknejad, E. Hashemizadeh, B. Basirat, Computational method based on Bernstein operational matrices for nonlinear Volterra-Fredholm-Hammerstein integral equations, Commun. Nonlinear. Sci. Numer. Simulat. 17 (2012) 52-61.

[39] D. Rostamy, K. Karimi, Bernstein polynomials for solving fractional heat- and wave-like equations, Fract. Calc. Appl. Anal. 15 (2012) 556-571. 
[40] S.J. Liao, Ph.D. Thesis, Shanghai Jiao Tong University, (Shanghai, Chaina, 1992).

[41] S.J. Liao, Beyond perturbation: introduction to the homotopy analysis method, CRC Press, Boca Raton: Chapman Hall; 2003.

[42] S. Abbasbandy, Homotopy analysis method for heat radiation equations, International Communications in Heat and Mass Transfer 34 (2007) 380-387.

[43] J. Cheng, S.J. Liao, R.N. Mohapatra, K. Vajravelu, Series solutions of nano boundary layer flows by means of the homotopy analysis method, J. Math. Anal. Appl. 343 (2008) 233-245.

[44] S. Abbasbandy, Homotopy analysis method for generalized Benjamin-Bona-Mahony equation, Z. angew. Math. Phys. 59 (2008) 51-62.

[45] A. Sami Bataineh, M.S.M. Noorani, I. Hashim, On a new reliable modification of homotopy analysis method, Commun. Nonlinear Sci. Numer. Simulat. 14 (2009) 409-423.

[46] S. Abbasbandy, E. Babolian, M. Ashtiani, Numerical solution of the generalized Zakharov equation by homotopy analysis method, Commun. Nonlinear Sci. Numer. Simulat. 14 (2009) 4114-4121.

[47] S. Abbasbandy, T. Hayat, Solution of the MHD Falkner-Skan flow by homotopy analysis method, Commun. Nonlinear Sci. Numer. Simulat. 14 (2009) 3591-3598.

[48] M.M. Rashidi, S. Dinarvand, Purely analytic approximate solutions for steady three-dimensional problem of condensation film on inclined rotating disk by homotopy analysis method, Nonlinear Analysis: Real World Applications 10 (2009) 2346-2356.

[49] S. Abbasbandy, Homotopy analysis method for the Kawahara equation, Nonlinear Analysis: Real World Applications 11 (2010) 307-312.

[50] S. Dinarvand, On explicit, purely analytic solutions of off-centered stagnation flow towards a rotating disc by means of HAM, Nonlinear Analysis: Real World Applications 11 (2010) 3389-3398.

[51] S. Abbasbandy, E. Shivanian, Prediction of multiplicity of solutions of nonlinear boundary value problems: Novel application of homotopy analysis method, Commun. Nonlinear Sci. Numer. Simulat. 15 (2010) 3830-3846.

[52] H. Jafari, M. Alipour, Solution of the Davey Stewartson equation using homotopy analysis method, Nonlinear Analysis: Modelling and Control 15 (2010) 4:423-433.

[53] A. Zare, M.A. Firoozjaee, Numerical solution for Maxwells equation in metamaterials by Homotopy Analysis Method, Journal of Mathematics and Computer Science 3 (2011) 2:225-235.

[54] H.M. Sedighi, K.H. Shirazi, J. Zare, An analytic solution of transversal oscillation of quintic non-linear beam with homotopy analysis method, International Journal of Non-Linear Mechanics 47 (2012) 777784. 
[55] R. Ellahi, M. Raza, K. Vafai, Series solutions of non-Newtonian nanofluids with Reynolds model and Vogels model by means of the homotopy analysis method, Mathematical and Computer Modelling 55 (2012) 1876-1891.

[56] M. Ghoreishi, A.I.B.Md. Ismail, A.K. Alomari, A.S. Bataineh, The comparison between Homotopy Analysis Method and Optimal Homotopy Asymptotic Method for nonlinear agestructured population models, Commun. Nonlinear Sci. Numer. Simulat. 17 (2012) 1163-1177 .

[57] F. Guerrero, F.J. Santonja, R.J. Villanueva, Solving a model for the evolution of smoking habit in Spain with homotopy analysis method, Nonlinear Analysis: Real World Applications 14 (2013) 549-558.

[58] R. Ellahi, The effects of MHD and temperature dependent viscosity on the flow of non-Newtonian nanofluid in a pipe: Analytical solutions, Applied Mathematical Modelling 37 (2013) 1451-1467.

[59] Touqeer Nawaz, Ahmet Yildirim, Syed Tauseef Mohyud-Din, Analytical solutions ZakharovKuznetsov equations, Advanced Powder Technology 24 (2013) 252-256.

[60] M. Shaban, S. Kazem, J.A. Rad, A modification of the homotopy analysis method based on Chebyshev operational matrices, Mathematical and Computer Modelling (In Press).

[61] J. Saeidian, Sh. Javadi, Some Notes on the Convergence Control Parameter in the Framework of the Homotopy Analysis Method, Journal of Mathematics and Computer Science 9 (2014) 2:103-110.

[62] Y. Khan, A. Hussain, N. Faraz, Unsteady linear viscoelastic fluid model over a stretching/shrinking sheet in the region of stagnation point flows, Scientia Iranica (In Press).

[63] C. Canuto, M. Y. Hussaini, A. Quarteroni, and T. A. Zang, Spectral methods in fluid dynamics, Springer-Verlag, New York, 1988. 